\title{
APROXIMACIONES DE INTERVENCIÓN POLÍTICA DESDE LAS RAMAS DE PODER PÚBLICO PARA LA SUPERACIÓN INTEGRAL DE LA POBREZA EN AMÉRICA LATINA
}

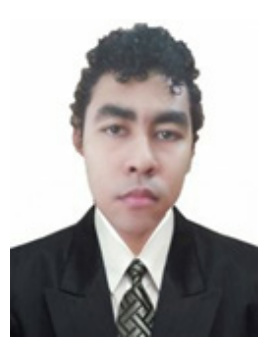

Abel Antonio Diaz Castellar

Institución Educativa Los Andes, Secretaria de Educación de Antioquia, Colombia

abel.diaz@ielosandeschigorodo.edu.co

DOI: 10.37594 /oratores.n13.415

Fecha de aceptación:28/10/2020

\section{RESUMEN}

El presente ensayo se realizó con el objetivo de plantear aproximaciones de intervención política desde las ramas de poder público para la superación integral de la pobreza en América Latina. Para ello se utilizó la metodología cualitativa, con técnica de análisis documental, mediante la revisión bibliográfica de bases de datos científicas, tales como Dialnet, Scielo, Redalyc, Researchgate y Google Scholar, previo ingreso de las siguientes expresiones en sus motores de búsqueda: como superar la pobreza en América Latina, intervención política a la pobreza y causas de la pobreza en América Latina. Como criterios de calidad, se tuvo en cuenta: la antigüedad (artículos no mayores a diez años desde su publicación), pertinencia del contenido y relevancia (credibilidad y transferibilidad), por el número de citaciones de otros autores. Como resultado, las aproximaciones de intervención política propuestas consisten en la unificación política de los países de América Latina y la definición de un Sistema Económico Regional, con ajustes a las funciones de las ramas del poder público. Así, en el poder legislativo, se propone el nombramiento por meritocracia y democracia de los dirigentes. En el poder ejecutivo, se proponen estrategias en cada ministerio que lo estructura, según su función. Por último, en el poder judicial, frente a delitos, además de la privación de la libertad, se proponen medidas formativas y de servicio comunitario. En conclusión, desde una mirada crítica, aunque las anteriores propuestas son necesarias, no son suficientes; adicionalmente, sus actores, deben ser motivados por principios de honestidad y ética profesional.

Palabras clave: Superación, Pobreza, América Latina, Poder Público, Sistema Económico Regional, Socio-empleado. 


\section{POLITICAL INTERVENTION APPROACHES FROM THE BRANCHES OF PUBLIC POWER FOR THE COMPREHENSIVE OVERCOMING OF POVERTY IN LATIN AMERICA ABSTRACT}

The present essay was carried out with the objective of proposing political intervention approaches from the branches of public power for the comprehensive overcoming of poverty in Latin America. For this, the qualitative methodology was used, with a document analysis technique, through the bibliographic review of scientific databases, such as Dialnet, Scielo, Redalyc, Researchgate and Google Scholar, after entering the following expressions in their search engines: as overcoming poverty in Latin America, political intervention to poverty and causes of poverty in Latin America. As quality criteria, the following were taken into account: seniority (articles not older than ten years since their publication), relevance of the content and relevance (credibility and transferability), by the number of citations from other authors. As a result, the proposed political intervention approaches consist of the political unification of the Latin American countries and the definition of a Regional Economic System, with adjustments to the functions of the branches of public power. Thus, in the legislative power, the appointment by meritocracy and democracy of the leaders is proposed. In the executive branch, strategies are proposed in each ministry that structures it, according to its function. Finally, in the judiciary, in the face of crimes, in addition to deprivation of liberty, training and community service measures are proposed. In conclusion, from a critical point of view, although the previous proposals are necessary, they are not enough; additionally, its actors must be motivated by principles of honesty and professional ethics.

Keywords: Overcoming, Poverty, Latin America, Public Power, Regional Economic System, Socio-employee.

\section{INTRODUCCIÓN}

El presente ensayo se compone de los siguientes apartados: identificación del problema, objetivo, antecedentes, síntesis de las tendencias teóricas en torno a la superación de la pobreza y propuesta de superación integral de la pobreza en América Latina. En primer lugar, en la identificación del problema se muestran cifras sobre el número de personas pobres en América Latina, la tendencia a la desigualdad, así como la meta trazada para superarla en los próximos diez años. En segundo lugar, se indica el objetivo del ensayo, a saber, plantear aproximaciones de intervención política desde las ramas de poder público para la superación integral de la pobreza en América latina. En tercer lugar, en los antecedentes se muestra el concepto de pobreza como un fenómeno político integral, que trasciende los ingresos económicos. En cuarto lugar, se realiza 
una síntesis de las tendencias teóricas frente a los intentos por superar la pobreza, las cuales tienen en común el abordaje unidimensional del fenómeno. En quinto lugar, se presentan los argumentos referentes a las aproximaciones de intervención política desde cada rama del poder público para la superación integral de la pobreza en América Latina. Por ultimo, en la conclusión se sostiene que los actores del cambio deben articular los conocimientos, experticia y habilidades disciplinares, con una conducta plenamente ética y de servicio, para garantizar la efectividad de dichas políticas y programas de intervención.

\section{IDENTIFICACIÓN DEL PROBLEMA}

En el día a día de los países de América latina y en general del mundo, existe una realidad que afecta a la mayoría de la población: la pobreza. De hecho, según investigaciones realizadas por Amarante, Galván y Mancero (2016) y Bonaglia, Parra y Zamora (2020), en América Latina la desigualdad y pobreza tiene grandes números: 220 millones de personas son pobres y se espera que con la situación de emergencia sanitaria causada por el covid-19, dicha cifra aumente en 35 millones. Como evidencia de lo anterior, en la siguiente tabla se muestra el alto índice de desigualdad y pobreza alrededor de 2002 y 2012.

\section{Tabla 1. Estado de pobreza y desigualdad alrededor de 2002 y 2012 en América Latina}

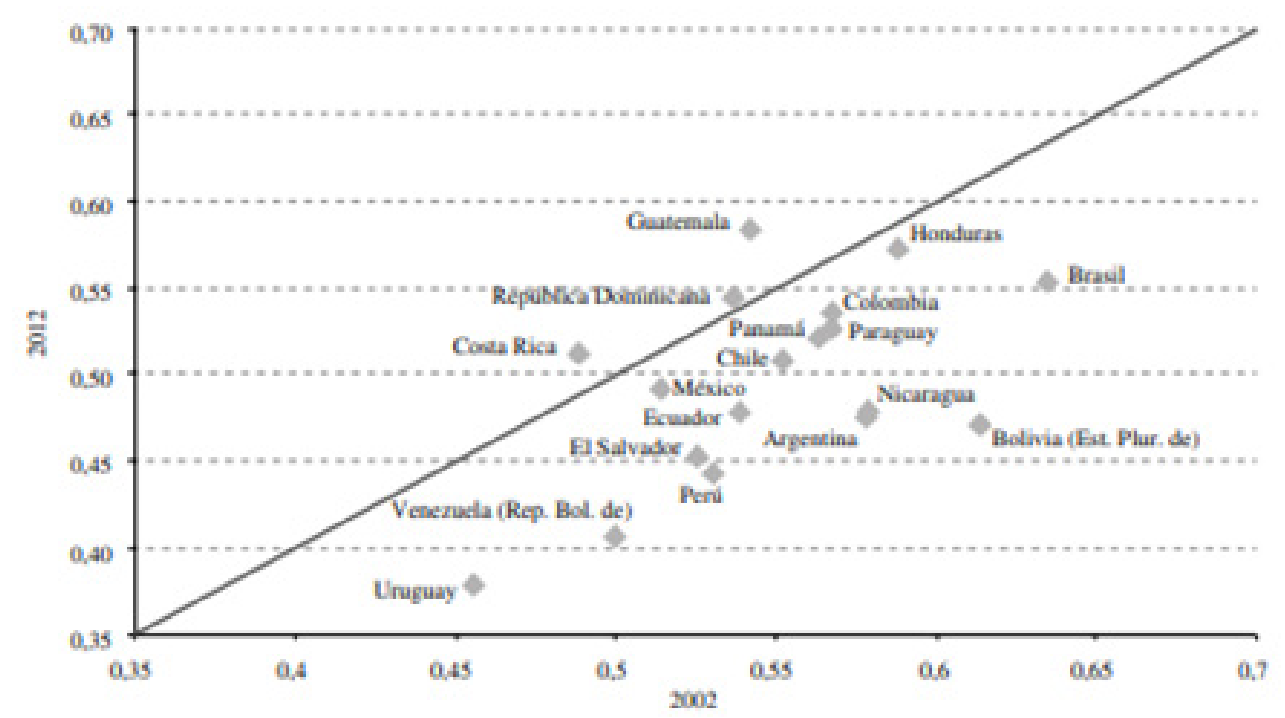

Nota: Los datos numéricos de la tabla corresponden al coeficiente de Gini.

Fuente: Amarante, Galván y Mancero (2016, p. 31).

Frente al análisis de la tabla anterior, cabe aclarar que el coeficiente de Gini es un número entre 0 y 1 , en donde 0 corresponde con la perfecta igualdad, es decir, todos tienen los mismos ingresos y donde el valor 1 se corresponde con la perfecta desigualdad. Por lo tanto, a más alejado esté un 
país del origen del plano, mas desigualdad y pobreza posee. Así, en 2002 y 2012, el panorama en materia de economía social, de los países de América latina, evidencia un notable índice de desigualdad y pobreza, índice, que seguramente se incrementará tras el impacto económico que causa la pandemia del covid-19.

Ahora bien, un sinnúmero de propuestas de solución frente a la superación de la pobreza, han girado en torno a programas y proyectos de tipo paliativo, sujeto a periodos de tiempo concretos (Max, Elizalde \& Hopenhayn 2010). Otras abordan el problema desde una perspectiva de etiqueta de gobierno, pensando que con autoproclamarse capitalistas, socialistas o mixtas, van a encontrar la solución. Sin embargo, diferentes autoridades en el tema insisten en que las propuestas que se han gestado al respecto, omiten atacar la pobreza como fenómeno multisistémico, con causas en el funcionamiento y estructura del tipo de gobierno, sea cual sea su denominación (Max, Elizalde \& Hopenhayn 2010; Rodríguez \& Sánchez, 2017).

Frente a lo anterior, en los Objetivos de Desarrollo Sostenible y el Programa de las Naciones Unidas para el Desarrollo se estableció como meta real para el 2030 la superación de la pobreza mediante la intervención política (Apoyado, 2018). Por lo tanto, la pregunta problema que en este ensayo se plantea es: ¿Qué aproximaciones de intervención política, deben plantearse, desde las ramas del poder público, para la superación de la pobreza en América Latina?

\section{ANTECEDENTES}

En primer lugar, en una aproximación al concepto de pobreza, en este ensayo se asume la postura de Amartya Sen (citada en Vargas, León \& Martínez, 2017), quien la define como la falta de capacidad de producir o de realizar su potencial productivo. En este sentido, para la mencionada autora, una persona podría tener ingresos y, no obstante, ser pobre, pues, además de estos, tiene "la imposibilidad de alcanzar un mínimo de realización vital por verse privado de las capacidades, posibilidades, y derechos básicos para hacerlo" (p.32). Por lo tanto, ser pobre es una condición de carencia integral, por lo que se necesita una intervención de la misma naturaleza para superarla.

\section{Síntesis de las tendencias teóricas frente a la solución de la pobreza}

Dentro de las principales tendencias teóricas en torno a propuestas de mitigación o solución de la pobreza se tienen las siguientes: Teoría de Crecimiento Económico y de Goteo, Teoría de las Necesidades Básicas, Teoría del Desarrollo Humano, Teoría del Empoderamiento, y la Teoría de las Capacidades (Martínez, 2017).

Dichas teorías tienen en común el abordaje de la pobreza de manera unidimensional y desde 
planes de intervención concretos, en muchos casos, basados en concepciones de pobreza reducidas a necesidades básicas y no en la integralidad y lo multisistémico del fenómeno, referido por Amartya Sen ( citada en Vargas, León \& Martínez, 2017), además de Giunta y González (2020).

\section{Propuesta de superación integral de la pobreza en América Latina}

La propuesta de solución planteada en este ensayo consiste en plantear aproximaciones de intervención política consistentes en una reforma estructural de gobierno, que comienza con la unificación política de los países de América y las organizaciones al interior de este: el Mercado Común del Sur (MERCOSUR), Comunidad Andina de Naciones (CAN), El CARICOM (Comunidad del Caribe), La Unión de Naciones Suramericanas (UNASUR), El Sistema de la Integración Centroamericana (SICA), La Alianza Bolivariana para los Pueblos de Nuestra América (ALBA), Alianza del Pacífico (AdP), La Comunidad de Estados Latinoamericanos y Caribeños (CELAC), para potenciar las fortalezas y disminuir las debilidades de cada uno, en sus políticas, proyectos y planes destinados a superar integralmente la pobreza (Roldán \& Norato, 2014). Esta unificación implica que debe construirse la estructura directiva del nuevo estado, es decir, la conformación de las ramas del poder público: legislativo, ejecutivo y judicial, bajo el nombre de Estados Unidos Latinoamericanos y un Sistema Económico Regional.

En ese sentido, para generar condiciones de bienestar, desarrollo social, dignidad y calidad de vida de la sociedad latinoamericana, con el fin de superar integralmente la pobreza, uno de los primeros pasos consiste en garantizar, desde los representantes de todas las ramas del poder público, una cultura política de actuación ética, con transparencia, en ausencia y oposición a la corrupción, así como la formulación de políticas de tipo estructural, con base en un estudio exhaustivo previo en torno a la magnitud y aristas del problema en la región latina (Salama \& Valier, 1995; Pernalete, 2015). Asimismo se deben formular políticas directivas generales en torno a la superación integral de la pobreza, así como planes y proyectos específicos, que permitan materializar las políticas iniciales, mediante la aproximación progresiva entre su axiología, teleología y los resultados concretos tras su ejecución (Páez \& Ceballos, 2014).

En cuanto a la estructura de la propuesta, esta se desglosa en las diferentes ramas del poder público. Para el caso del poder legislativo, se propone la construcción colectiva de leyes en los Estados Unidos Latinoamericanos, conformación del Sistema Económico Regional, institucionalización de los Derechos Básicos de Consumo Ciudadano, estatus de socios-empleados y nombramiento de representantes, es decir, directores; e integrantes, es decir, socio-empleados, para promover la conducta ética y representatividad real del pueblo. 
Respecto al poder ejecutivo, se explica la propuesta en función de los diferentes ministerios, entre otros órganos que lo conforman, tales como el Ministerio de Hacienda, Ministerio de Defensa, Ministerio de Educación, Ministerio de Ciencia Tecnología e Innovación, Ministerio de Ambiente y Desarrollo Sostenible, Ministerio de Minas y Energía, Ministerio de Agricultura y Desarrollo Rural. Asimismo, en la argumentación se hace énfasis en las características de la ejecución y administración que posibilitan de manera viable y factible, la superación integral de la pobreza.

Por último, en la reforma al poder judicial, se argumenta la propuesta en función de la nueva administración de justicia que hace posible la percepción de justicia en la sociedad, la independencia de las demás ramas, como debe ocurrir con cada una de las ramas del poder público, y en la penalización de delitos, se propone complementar la privación de la libertad con medidas correctivas de tipo pedagógico para la no repetición de las conductas socialmente indeseadas y la formación de un ser humano apto para la vida y desarrollo comunitario, como característica de una sociedad que avanza hacia la superación integral de la pobreza.

\section{Reforma al poder legislativo}

En una aproximación a las funciones del poder legislativo, Hervia de la Jara (2010) plantea que esta rama del poder público se encarga de crear las leyes que expresan en un texto escrito una decisión política y consta de tres etapas: "iniciativa, que permite a diferentes actores presentar una propuesta; constitutiva, que transcurre específicamente dentro del parlamento; y la etapa integrativa de eficacia, que tiene que ver con los procedimientos del poder Ejecutivo para publicar la norma legal" (p.3).

En relación con los actores que presentan propuestas para su constitución legal, si se habla de un contexto democrático, se entiende que todos los distintos segmentos poblacionales, con sus concepciones e ideologías, deben participar en la construcción de leyes que fomenten los intereses y el bienestar común (Domínguez, 2012).

Ahora bien, dicha finalidad de bienestar social se ve truncada por el fenómeno de la corrupción, que consiste en el aprovechamiento de un cargo o función pública en beneficio de intereses privados, particulares o compartidos, reprobables por la sociedad, aunque pueden o no ser castigados por el código penal (Soriano, 2011; Bautista, 2012; Hechler, 2013).

Por lo anterior, en vista de los altos índices de corrupción desde los representantes de las ramas del poder público en América Latina, y la contradicción teleológica que representa en relación con la democracia, se propone un mecanismo de meritocracia y veeduría ciudadana a 
los representantes de las ramas del poder público (Lacoviello \& Strazza, 2011; Hechler, 2013), para aumentar la probabilidad escoger los individuos con los aspectos actitudinales y aptitudinales necesarios para construir desde las bases legales un sistema político-económico que propenda por el bienestar, confort y desarrollo del pueblo, entendiendo que dichas leyes no son un producto acabado sino que se desarrollan continuamente mediante el tránsito desde la conciencia social hasta la institucionalización de la norma legal.

Concretamente, la reforma propuesta al poder legislativo consiste en la modificación de las leyes que regulan el acceso, permanencia y gestión de los empleados de las ramas del poder público, por meritocracia y democracia para los representantes de dichos poderes, y con meritocracia para el resto de los integrantes, dado que quienes perciben un adecuado funcionamiento de la meritocracia, también perciben menos desigualdad social (Castillo, Torres, Atria \& Maldonado, 2019).

En primer lugar, se propone que para el acceso y permanencia, para acceder al cargo de representante(dirigente) o integrante(operario) en cada rama del poder público, bajo el criterio de la meritocracia, el ciudadano postulado deberá cumplir con el requisito mínimo de formación en para el cargo y experiencia relacionada. Además deberá superar una prueba aptitudinal de conocimientos de dimensión teórico-práctica, así como una prueba actitudinal de motivaciones, compromisos éticos y comportamientos esperados en el cargo, de dimensión práctica. Además, el caso de los aspirantes a dirigentes, únicamente los que aprueben serán vinculados al sistema económico-estatal, en estricto orden de mérito, según las vacantes. En el caso de los aspirantes a integrantes, deberán pasar por un programa educativo de refuerzo, y serán vinculados una vez adquieran satisfactoriamente las competencias de su cargo. Respecto a la permanencia en el cargo, estos serán evaluados con cierta periodicidad por su jefe inmediato y los demás empleados.

Adicionalmente, para el caso de los aspirantes a representantes de las ramas del poder público, una vez cumplidos los requisitos anteriores, deberán construir un plan de trabajo afín a su cargo, a disposición de los integrantes del sistema, para que puedan realizar los ajustes necesarios, orientados a la administración eficiente, con equidad, para el bienestar social, dignidad y confort. Dichos representantes deben comprometerse por escrito a cumplir lo pactado, con evidencias de los logros parciales, para la continuidad en su cargo. Posteriormente se someterá a la elección por democracia, y el representante electo, empezará en periodo de prueba, por lo menos un año.

En segundo lugar, en cuanto a la permanencia en el cargo, cada año será evaluado por un órgano independiente a las tres ramas del poder público, y por la ciudadanía en general a través de un espacio de veeduría, con base en los indicadores de acuerdo con lo pactado en la fase de campaña. 
Para el caso de los integrantes del poder legislativo, por ser este una rama que regula a las demás (Hervia de la Jara, 2010), además de la meritocracia, se debe garantizar la representatividad en la vos y concepciones de la sociedad. Para ello, se conformarán con líderes de diferentes regiones de América Latina, que recojan el sentir de sus comunidades, especialmente, las percepciones sobre los aspectos que les generan bienestar calidad de vida, dignidad y desarrollo.

\section{Reforma al poder ejecutivo}

Según Cadena y Gayosso (2016), en los estados democráticos el poder ejecutivo es el responsable de la gestión diaria del Estado, mediante la ejecución de políticas y leyes, y puede sugerir respecto a estas, en representación de la voluntad popular a la cual representa y defiende. Para ello posee una estuctura organizacional, compuesta, entre otros órganos, del Ministerio de Hacienda, Ministerio de Defensa, Ministerio de Educación, Ministerio de Ciencia Tecnología e Innovación, Ministerio de Ambiente y Desarrollo Sostenible, Ministerio de Minas y Energía, Ministerio de Agricultura y Desarrollo Rural.

No obstante, numerosas investigaciones refieren que en América Latina, lejos de ser garantes de la voluntad popular, responden a intereses de algunos sectores reducidos, incluso, toman decisiones que ponen en tela de juicio su idoneidad y contribuyen a la reproducción y producción de situaciones de pobreza para la mayoría de la población (Stampini, Robles, Sáenz, Ibarrarán \& Medellín, 2015; Álvarez, 2017). Por esta razón en este apartado se realizan propuestas de reforma del poder ejecutivo, desde los ministerios que lo estructuran, con el fin de propiciar la superación integral de la pobreza.

\section{En el Ministerio de Hacienda}

Según Marín y Plazas (2019), la secretaría de hacienda está encargada de "la prestación de los servicios públicos esenciales, para garantizar a la sociedad condiciones de dignidad humana, armonía y paz” (p.1). Además, según el mismo autor, "de su adecuado manejo depende el nivel de bienestar que se puede suministrar a la población, por lo que se constituye como fundamento esencial de la política económica para la estabilidad de una nación” (Marín \& Plazas, 2019, p.2).

Así para lograr la finalidad de dignidad, armonía, paz y desarrollo social, no es un secreto que por décadas, el modelo de administración de los recursos es esencialmente capitalista, y el objetivo que ha logrado ha sido precisamente el contrario, la acumulación excesiva de recursos en unos pocos, a costa del detrimento en las condiciones de dignidad y bienestar social en todos los sentidos (Borón, 2008), por lo que actualmente, distintos autores se refieren a dicho modelo como inviable para el desarrollo y superación de la pobreza en América Latina (Borón, 2008; Ancada, 
2008; Martínez, 2008; Harnecker, 2010; Fernández, 2014). Asimismo, las nuevas tendencias en la administración de los recursos, se orientan a modelos más democráticos, institucionales, con aproximaciones socialistas, donde todos los integrantes del sistema económico (ciudadanos) son socios y a la vez empleados del sistema, con voz y voto para decidir y ejecutar (Martínez, 2008; Harnecker, 2011).

De esta manera, se propone un cambio de la figura de presidente a administrador nacional, con una estructura directiva subsiguiente, dotada de empleados-socios de la gran empresa estatal en América Latina, los cuales tendrán acciones del patrimonio nacional correspondiente y en el macroproyecto económico, lo que maximizará el beneficio obtenido (Briceño, 2013). Además, de acuerdo a su cargo, funciones, responsabilidades en la estructura jerárquica, el salario de los sociosempleados podrá variar ligeramente. No obstante, aun el más salario bajo, deberá ser de tal cuantía que permita amplias condiciones de bienestar social, salud, previo un estudio de percepciones individuales relacionadas.

Por lo tanto, en lo relacionado con el ministerio de hacienda, en este ensayo se propone la democratización de los recursos económicos y medios de producción de América Latina, es decir, renombrarlos como parte del erario estatal y ser utilizados por los representantes de la rama del poder ejecutivo o administración nacional, para promover el desarrollo económico, en dos grandes vertientes: la seguridad de consumo nacional y la elaboración de productos del sector económico secundario y/o terciario para exportación (Schmidtke, Koch \& García, s.f). Dichas actividades económicas son factibles de desarrollarse en América Latina, pues esta región presenta diversidad de estos sectores económicos entre países, lo que permite la complementariedad económica al momento de fusionar sus actividades comerciales (Schmidtke, Koch \& García, s.f; Rodríguez \& Vanegas, 2014).

En consonancia con lo anterior, la actividad comercial de la macroempresa estatal consistirá en la exportación de sus productos y en la creación de multinacionales, pues este uno de los factores más importantes para el crecimiento económico y del PIB regional (Armijos, Ludeña \& Ramos, 2017). Además, muchos países latinoamericanos poseen empresas multinacionales, lo cual es un buen punto de partida, para fortalecer el comercio exterior (Casanova, 2015).

Ahora bien, luego del proceso de comercialización internacional, se destinará un fondo para los costos y gastos de producción y la utilidad será repartida equitativamente entre los miembros de la gran empresa América Latina, la cual se constituirá en su pago. No obstante, estos dineros serán utilizados solamente para actividad comercial externa, no habrá necesidad de mercado interno, 
pues las necesidades básicas estarán cubiertas gratuitamente por el hecho de ser socio-empleados del sistema y para el caso de los bienes adicionales que los ciudadanos deseen, deberán importarlos (Martínez, 2008; Max, Elizalde \& Hopenhayn, 2010; Harnecker, 2011).

Asimismo, se destinará un fondo para el ahorro, con el fin de atender sucesos imprevistos negativos que pueda afectar la economía de la región, tales como una catástrofe ambiental o biológica. De esta manera no solo se fortalece la economía desde la diversificación de sus fuentes y diferentes niveles de actividades económicas, sino que se logra mejorar la competitividad con los otros países, los ingresos y poder adquisitivo de los ciudadanos.

\section{En el Ministerio de Salud}

Según Cotlear et al. (2015), el ministerio de salud tiene como objetivo principal la formulación, ejecución, y evaluación de las políticas públicas relacionadas con la salud de los ciudadanos y riesgos profesionales, con el fin de que estos ejerzan su derecho a la protección a la salud.

No obstante, son muchos los investigadores que coinciden en que el servicio de salud en América Latina es precario (Martínez et al., 2011; Laurell, 2016; Báscolo \& Del Riego, 2018). A esto se le suma la alta percepción desfavorable de la ciudadanía, quienes explican la precariedad por la prioridad de intereses económicos empresariales por encima del bienestar ciudadano (Sojo, 2011).

Por lo anterior, se propone una reestructuración al sistema de salud, empezando por sustituir el concepto de servicio de salud, por derecho a la salud. Este derecho a la salud será garantizado por el área de gestión del Sistema Económico Regional, encargada del cubrimiento de necesidades básicas de salud de los socio-empleados de sistema económico estatal, de forma completamente gratuita.

Para ello se deben reestructurar las empresas prestadoras de salud, en el sentido de mejorar la infraestructura y capacidad instalada de los hospitales, y garantizar con celeridad la atención hospitalaria pertinente según la complejidad de cada caso. Además, la prestación de este servicio se distribuirá a cargo de tantas sucursales como sea necesario, cada una con un número de ciudadanos que atender, establecido con base en estudios piloto. Además, la calidad y cobertura del servicio prestado por el área de gestión de salud del Sistema Económico Regional, serán evaluadas y sujetas a planes de mejoramiento, con base en las percepciones evidentes a través de espacios de veeduría ciudadana. 


\section{En el Ministerio de Defensa}

Según Eissa (2017), el ministerio o secretaría de defensa tiene como objetivo la planeación, organización y administración de programas y proyectos, con el objetivo de defender la soberanía de cierta nación, mantener el orden constitucional y convivencia democrática.

De ese modo, la propuesta en este ministerio consiste en convocar a las fuerzas armadas, legales e ilegales a un cese bilateral del fuego, con el fin de establecer un acuerdo de paz que incluya reparación a víctimas, resocialización, educación para la resolución pacífica de conflictos, convivencia ciudadana y educación para el ingreso al Sistema Económico Regional. Luego, serán inscritos en la planta de socios-empleados del mencionado sistema económico previo cumplimiento de los requisitos meritocráticos para ello. Solamente quedará un grupo reducido de la fuerza pública, para defender la soberanía, integridad regional y velar por la convivencia ciudadana.

\section{En el Ministerio de Educación}

Según Vásquez (2015) el Ministerio de Educación se encarga de elaborar la política nacional de educación, lo que incluye criterios y parámetros que permitan el acceso, permanencia y calidad en todos los niveles de formación.

Frente a la calidad educativa, algunos autores manifiestan que, salvo en algunos casos, la educación en América Latina, se orienta a los aprendizajes teóricos, es decir, el aprender a conocer y, en menor medida, al aprender a hacer (Rivas, 2015; Plá, 2016). Por tanto, se propone una reforma educativa que articule en su currículo los cuatro pilares de la educación, es decir, el aprender a conocer, aprender a hacer, aprender a convivir juntos y aprender a ser, contextualizados con el nuevo sistema económico, en virtud del perfil esperado de los ciudadanos en calidad de sociosempleados de dicho sistema (Delors, citado en Garcés, Monsalve, Chavarriaga \& Moreno, 2017). En ese sentido, se debe realizar una transposición didáctica del saber implicado en todo el proceso productivo y de desarrollo económico, con el fin de convertirlo en un saber enseñable, enfocado en los mencionados cuatro pilares de la educación.

De esta manera, la educación se pretende desarrollar con base en el proyecto de vida nacional, desde la actividad económica comercial conjunta y los valores que se requieren para la convivencia, formación y autorrealización personal, sentido de curiosidad e investigación, conciencia ética, de acuerdo a las necesidades de la cultura y forma de vida, producto de la transformación de las ramas del poder público, especialmente la referida al nuevo sistema económico nacional para que los estudiantes, los cuales serán los socios empleados del mañana, hereden y continúen desarrollando su estado de bienestar, confort y participación ciudadana en la dirección y organización del estado 
regional propuesto.

Para lograr lo anterior, se propone construir un Currículo Unificado Regional cuya finalidad sea el aprendizaje conceptual, procedimental, crítico de las diferentes aristas de las ramas del poder público, así como el proceso productivo del sistema económico regional, de menor a mayor complejidad, según el nivel de formación y la formación de valores éticos, para potenciar los primeros aprendizajes y garantizar la continuidad y progreso del nuevo sistema implementado. Así, en el pilar educativo aprender a conocer, esto implica reconstruir los contenidos y el discurso pedagógico subyacente en las áreas de conocimiento que comúnmente se abordan, en consonancia con el nuevo currículo unificado regional (Plá, 2016).

Además del aprendizaje conceptual, es necesario que la nueva educación potencie el aprender a hacer (Delors, citado en Garcés et al., 2017), mediante la aplicación y puesta en contexto especialmente en actividades propias del Sistema Económico Regional, según el nivel de formación en que se encuentren los estudiantes.

Ahora bien, no bastará solo con aprender a conocer y hacer, en tanto se constituyen solo en un ejercicio de reproducción de conocimiento que pertenece a la cultura. Por esta razón, se resalta educar en el ser (Delors, citado en Bécart \& Garrido, 2016), en primer lugar desde una perspectiva disciplinar, para la adquisición de habilidades tales como la metacognición, pensamiento críticoreflexivo, investigación; y en segundo lugar, desde una perspectiva comportamental, para la formar una adecuada conciencia ética laboral, profesional, responsabilidad ambiental, así como el manejo de finanzas personales, y habilidades para llevar una vida sana y productiva individual y en sociedad.

Por último, es necesario formar en el aprender a vivir juntos (Delors, citado en Garcés et al., 2017), es decir, construir escenarios de aprendizaje para la convivencia ciudadana, las buenas relaciones interpersonales, valores morales en relación con el otro, que propendan en el desarrollo y fortalecimiento de la convivencia pacífica.

\section{En el Ministerio de Ciencia, Tecnología e Innovación}

Según López (2017), este ministerio se encarga de ejecutar las políticas relacionadas con ciencia, tecnología e innovación productiva, para incorporar el conocimiento y la innovación como base de su desarrollo social y económico.

A pesar de la claridad conceptual de su función, diferentes autores manifiestan que, en América Latina, a este ministerio no se le da la debida importancia: existe un bajo porcentaje del PIB en 
la asignación de recursos y acompañamiento a los procesos de desarrollo científico (Lemarchand, 2016; Baptista, 2018). Por lo tanto, se propone una reforma al ministerio de ciencia tecnología e innovación, para integrarlo en el sistema económico estatal, el cual, mediante un proceso de articulación con el ministerio de educación regional, se encargará de promover la investigación, para la construcción y generación de nuevo conocimiento que permita el mejoramiento continuo del sector productivo, sea para potenciar los procesos ejecutados, o para generar nuevas formas de producción.

Es especialmente importante hacer énfasis en estas nuevas formas de producción, pues dichos constructos se constituirán en modalidades alternativas para afrontar con éxito contigencias biológicas como la que ocurre actualmente con el covid-19. Por ejemplo, podrían fomentarse investigaciones para establecer una economía regional basada en el comercio internacional de activos electrónicos. Por tanto, será competencia de este ministerio fortalecer la ciencia tecnología e innovación en todas y cada una de las etapas por las que atraviesa el proceso empresarial, que permita el desarrollo de nuevas ideas de comercio internacional viables y factibles. Asimismo, este ministerio impulsará en la misma medida el desarrollo humano mediante la aplicación de la ciencia, tecnología e innovación, para mejorar de la calidad de vida y bienestar de los ciudadanos, desde la satisfacción de las necesidades básicas hasta las socialmente complejas. Por último, se enfatiza que este ministerio será fundamental en la promoción de la investigación, ciencia y tecnología para el mejoraramiento organizacional y disciplinar de los demás ministerios.

\section{En el Ministerio de Agricultura y Desarrollo Rural}

Según Sabourin, Massardier y Sotomayor (2016), este ministerio tiene como objetivos primordiales la formulación, coordinación y adopción de las políticas, planes, programas y proyectos del sector agropecuario, esquero, así como otras actividades del sector primario, no asociadas al Ministerio de Minas y Energía.

Aunque las políticas de este ministerio han anunciado desde el discurso el estudio y la promoción de las agriculturas familiares en una perspectiva de desarrollo rural mediante un modelo inclusivo generando empleos, mayor autonomía y sustentabilidad de la producción y reducción de la pobreza y de las desigualdades con intervención de la Comisión Económica para América Latina y el Caribe (CEPAL) en realidad las políticas han sido débiles y han aumentado la brecha entre el campo y la urbanidad (Botella \& Suárez, 2016).

Frente a lo anterior, este ministerio se encargará de fortalecer y ejecutar las políticas, programas y proyectos destinados a maximizar la producción de los recursos agropecuarios de 
los que cada socio-empleado tiene acciones, pues estos se constituyen en materia prima para la elaboración de productos económicos industriales y en forma de bienes y servicios para comercio internacional, así como para la seguridad alimentaria regional, bajo el principio de sostenibilidad ambiental. En síntesis, la propuesta se basa en la asignación de acciones equitativas del patrimonio nacional, para todos los ciudadanos, incluidos los campesinos, con una educación que les permita realizar actividades para el desarrollo del sistema económico regional y que contribuyan a la vez, en el beneficio y calidad de vida personal.

\section{En el Ministerio de Minas y Energía}

Según Hincapié (2017), el Ministerio de Minas y Energía tiene la función de administrar los recursos naturales no renovables del país, para su uso racional en función de las necesidades y la protección del medio ambiente, con el fin de garantizar su conservación en el tiempo.

En concordancia con lo anterior, por tratarse de recursos finitos, se propone su uso exclusivamente para consumo interno, para prolongar su aprovechamiento en el tiempo; para ello estará en articulación con el Ministerio de Ciencia, Tecnología e Innovación y el Ministerio de Ambiente y Desarrollo Sostenible para el desarrollo de mecanismos que permitan el aprovechamiento de fuentes de energía alternativas. Asimismo, su proceso de extracción se debe hacer con el principio de sostenibilidad socio-ambiental, para lo cual, estará en articulación con el Ministerio de Ambiente y Desarrollo Sostenible. Para finalizar, los productos obtenidos de los recursos no renovables, por tratarse de consumo interno, serán distribuidos de forma gratuita para todos los socio-empleados pertenecientes al Sistema Económico Regional.

\section{En el Ministerio de Ambiente y Desarrollo Sostenible}

Según Rodríguez y Espinoza (2016), la función de este ministerio es garantizar el uso racional de los recursos ambientales a través de normas y directrices en materia de recursos renovables y no renovables, para su conservación en el tiempo.

Por lo tanto, dentro de la gestión de este ministerio se propone explotar los recursos naturales, especialmente los no renovables, en sujeción a un inventario de consumo, para estimar la cantidad y el tiempo de aprovechamiento. Asimismo, es urgente elaborar, en articulación con el Ministerio de Minas y Energía y el Ministerio de Ciencia, Tecnología e Innovación, proyectos y programas para el aprovechamiento de fuentes de energía alternativas y amigables con el ambiente, tales como la eólica, hídrica, solar, eléctrica, etc., en articulación con el ministerio de ciencia, tecnología e innovación, para que las generaciones futuras tengan disponibilidad de recursos energéticos. 
Asimismo, la densidad poblacional de las generaciones futuras también serán controladas con base en estudios de proyección de crecimiento económico y estado del patrimonio y PIB nacional, para estimar una tasa de crecimiento poblacional y la máxima cantidad de hijos por pareja, con el fin de no comprometer la estabilidad, sostenibilidad ni calidad de vida de los Estados Unidos Latinoamericanos.

\section{Reforma al poder judicial}

Según Sutil (2016), es una de las tres ramas del poder público, encargada de impartir Justicia en la sociedad, mediante la aplicación de las normas y principios jurídicos en la resolución de conflictos.

No obstante, existe una alta percepción ciudadana sobre la independencia de esta rama respecto a las otras, pues cuando los delitos son cometidos por altos funcionarios, tienden a quedar en la impunidad (Popkin, 2016). Por lo tanto, aunque es sabido que las ramas del poder público son independientes, se propone que a este ministerio se le aplique veeduría ciudadana, en aras de garantizar la intersubjetividad y el acuerdo colectivo para la imparcialidad en la administración de la justicia con celeridad. En ese sentido, se espera que la administración imparcial y con ausencia de impunidad, se constituya en objeto de aprendizaje de la ciudadanía y disminuya la incidencia delictiva (Popkin, 2016). Esto permitirá generar, poco a poco, un ambiente de confort, bienestar y seguridad social, los cuales son aspectos que caracterizan una condición social contraria a la pobreza.

Adicionalmente, se propone que el poder judicial apoye al ministerio o secretaría de defensa mediante el nombramiento de jueces de paz en microambientes, previo proceso de formación pertinente, con el fin de resolver conflictos rutinarios, pero que potencialmente puedan trascender. En el caso que esté implicado un delito, se debe aplicar una pena que, además de privar de la libertad, implique un proceso correctivo de formación pedagógica y la posterior prestación de servicio comunitario, para resarcir el delito cometido, con supervisión de funcionarios de la fuerza pública. En caso de asesinato, la pena para el victimario consistirá en trabajos de índole sociocomunitario y subsistirán únicamente con sus Derechos Básicos de consumo ciudadano.

\section{CONCLUSIÓN}

En este ensayo se plantéo una reforma estructural de gobierno fundamentada en la superación de la pobreza como factor político estructural y multidimensional. Por esta razón, se propone una alianza política de los países de América Latina, llamada los Estados Unidos Latinoamericanos, y la resignificación de las ramas del poder público, empezando por la independencia política efectiva de 
las mismas y culminando con la creación de políticas efectivas, es decir, aquellas que son evaluadas con base en el grado de cubrimiento de necesidades, pues la finalidad de dichas políticas, más que ejecutar obras, de ser la satisfacción de necesidades sociales.

Para finalizar, cabe destacar que toda política, planes y programas elaborados con el fin de mitigar o acabar con la pobreza, requieren de la articulación de competencias disciplinares y comportamentales. No obstante, de manera accidental o deliberada, en la sociedad se da a entender que el éxito de las mencionadas políticas, planes de desarrollo, etc. dependen en su mayoría, del conocimiento, experticia, experiencia en el campo, a la vez que se omiten las habilidades del ser, tales como honestidad y ética profesional, que moldean y le dan sentido a dichas competencias disciplinares. Esto es especialmente importante comprenderlo, ya que por mucha experticia y conocimiento que un funcionario público tenga, si su ética profesional no es la adecuada, no será efectivo plenamente en la finalidad de sus funciones.

Dicho de otro modo, la definición de una política disciplinar para solucionar la pobreza es una condición necesaria, pero no suficiente. La suficiencia se alcanza con la articulación de Ahora bien, aunque para nadie es un secreto la importancia de la honestidad y ética profesional, no es un tema del que se hable a menudo en la política; por el contrario, permanece bajo la sombra, oculto, como si su presencia no pareciera importarle a dichos actores. Por ello, cabe preguntarse: ¿por qué no le interesaría a un gobierno hablar de ética?, ¿por qué no se le da la importancia adecuada en las políticas implementadas?, ¿Acaso no le interesa que las políticas sean efectivas contra la pobreza?, ¿Será que sus verdaderas intenciones políticas distan de las acciones visibles y su discurso social? Hasta tanto no se respondan estas cuestiones y se asuman con la seriedad e importancia debida en el ejercicio político, para asegurar la adecuada motivación-acción de los dirigentes; probablemente la solución definitiva a la pobreza seguirá siendo escurridiza.

\section{REFERENCIAS BIBLIOGRÁFICAS}

- Amarante, V., Galván, M., \& Mancero, X. (2016). Desigualdad en América Latina: una medición global. Revista Cepal.

- Apoyado, E. (2018). Programa de las Naciones Unidas para el Desarrollo.

- Baptista, B. (2018). Una aproximación a las capacidades de diseño e implementación de políticas de ciencia, tecnología e innovación en América Latina. Revista Iberoamericana de Ciencia, Tecnología y Sociedad-CTS, 13(38).

- Barreda, M. (2011). La calidad de la democracia: Un análisis comparado de América Latina. Política y gobierno, 18(2), 265-295.

- Báscolo, E., Houghton, N., \& Del Riego, A. (2018). Lógicas de transformación de los 
sistemas de salud en América Latina y resultados en acceso y cobertura de salud. Revista Panamericana de Salud Pública, 42, e126.

- Bautista, O. D. (2012). El problema de la corrupción en América Latina y la incorporación de la ética para su solución. Espacios públicos, 15(35), 48-62.

- Bécart, A., \& Garrido, J. D. R. (2016). Fundamentos del coaching educativo: caracterización, aplicaciones y beneficios desde los cuatro pilares del saber. Plumilla educativa, 18(2), 344362 .

- Bécart, A., \& Garrido, J. D. R. (2016). Fundamentos del coaching educativo: caracterización, aplicaciones y beneficios desde los cuatro pilares del saber. Plumilla educativa, 18(2), 344362.

- Blanco, O. R., \& Sam, O. R. F. (2014). Teoría del bienestar y el Óptimo de Pareto como problemas microeconómicos. REICE: Revista Electrónica de Investigación en Ciencias Económicas, 2(3), 217-234.

- Bonaglia, F., Parra, S. N., \& Zamora, J. J. V. (2020). Una mirada al futuro post-COVID-19: hacia un nuevo pacto social en América Latina y el Caribe. Análisis Carolina, (21), 1.

- Botella, C., \& Suárez, I. (2016). Innovación para el desarrollo en América Latina: Una aproximación desde la cooperación internacional.

- Briceño Ruiz, J. (2013). Ejes y modelos en la etapa actual de la integración económica regional en América Latina. Estudios Internacionales (Santiago), 45(175), 9-39.

- Burchardt, H. J. (2017). La crisis actual de América Latina: causas y soluciones. Nueva Sociedad, (267), 114.

- Cadena, A. E., \& Gayosso, R. S. (2016). Las facultades constitucionales del Ejecutivo en América Latina: entre la concentración y la dispersión de poder. Estudios políticos, 37, 111141.

- Casanova, L. (2015). Las multinacionales latinoamericanas ante la nueva realidad. Familias empresarias y grandes empresas familiares en América Latina y España: Una visión de largo plazo, 71-94.

- Castillo, J. C., Torres, A., Atria, J., \& Maldonado, L. (2019). Meritocracia y desigualdad económica: Percepciones, preferencias e implicancias. Revista Internacional de Sociología, 77(1), 117

- Domínguez, J. (2012). Conflictos territoriales y democracia en América Latina. Universidad de Belgrano, Siglo Veintiuno.

- Eissa, S. (2017). Defensa Nacional: consideraciones para un enfoque analítico. Relaciones Internacionales, 26(53), 246-265.

- Fach Gómez, K. (2017). Inversiones internacionales y corrupción en América Latina: La función del arbitraje de inversiones en el avance de la institucionalidad democrática 
(International Investments and Corruption in Latin America: The Role of Investment Arbitration in the Advancement of Democracy). Max Planck Institute for Comparative Public Law \& International Law (MPIL) Research Paper, (2017-23).

- Fernández, Á. A. H. (2014). El socialismo del siglo XXI en América Latina: Características, desarrollos y desafíos. Revista de relaciones internacionales, estrategia y seguridad, 9(1), 131-154.

- Garcés, L. M. C., Monsalve, P. I. H., Chavarriaga, C. P., \& Moreno, J. A. T. (2017). Pilares de la educación inicial: mediadores para el aprendizaje. JSR Funlam Journal of Students' Research (histórico), (2), 86-94.

- Girón, A. (2016). Objetivos del desarrollo sostenible y la agenda 2030: Frente a Las Políticas públicas y los cambios de gobierno en américa latina. Problemas del desarrollo, 47(186), 3-8.

- Harnecker, M. (2010). El nuevo modelo económico del socialismo del siglo XXI. Algunos elementos para la discusión. SEMPLADES Los nuevos retos de América Latina. Socialismo y Sumak Kawsay. Quito: SEMPLADES, 77-90.

- Hechler, H. (2013). La UNCAC en breve. Una guía breve a la Convención de las Naciones Unidas contra la Corrupción para personal de embajadas y agencias donantes. U4 Brief.

- Hincapié, S. (2017). Entre el extractivismo y la defensa de la democracia. Mecanismos de democracia directa en conflictos socioambientales de América Latina.

- Iacoviello, M., \& Strazza, L. (2011). De partidocracias rígidas a meritocracias flexibles en América Latina. Documentos y aportes en administración pública y gestión estatal, 11(16), $51-95$.

- Laurell, A. C. (2016). Las reformas de salud en América Latina: procesos y resultados. Cuadernos de Relaciones Laborales, 34(2), 293.

- Lemarchand, G. A. (2016). Sistemas nacionales de ciencia, tecnología e innovación en América Latina y el Caribe.

- López, M. P. (2017). La cooperación en ciencia y tecnología entre Argentina y los países de América Latina. El caso del Ministerio de Ciencia, Tecnología e Innovación Productiva (2007-2015)

- López, M. P. (2017). La cooperación en ciencia y tecnología entre Argentina y los países de América Latina. El caso del Ministerio de Ciencia, Tecnología e Innovación Productiva (2007-2015)

- Marin, S. D. A., \& Plazas, J. D. C. (2019). Descentralización fiscal y finanzas públicas territoriales: análisis para el municipio de Pereira. CÓMITE EDITORIAL, 2.

- Martínez Rangel, R., \& Reyes Garmendia, E. S. (2012). El Consenso de Washington: la instauración de las políticas neoliberales en América Latina. Política y cultura, (37), 35-64.

- Martínez Ríos, B. (2017). Pobreza, discapacidad y derechos humanos. 
- Martínez, S., Carrasquilla, G., Guerrero, R., Gómez-Dantés, H., Castro, V., Arreola-Ornelas, H., ... \& Evans, R. (2011). Cobertura efectiva de las intervenciones en salud de América Latina y el Caribe: métrica para evaluar los sistemas de salud. salud pública de méxico, 53, s78-s84.

- Max-Neef, M., Elizalde, A., \& Hopenhayn, M. (2010). Desarrollo a escala humana: una opción para el futuro--Primera parte. Relectura de la crisis latinoamericana.

- Páez, C. C. T., \& Ceballos, G. G. (2014). Las políticas públicas y su papel en la gestión del desarrollo local. Revista Caribeña de Ciencias Sociales, (2014_04).

- Pernalete, M.E. (2015). Una reflexión acerca de la pobreza y la salud. Salud de los Trabajadores, 23(1), 59-61. Recuperado de: https://dialnet.unirioja.es/descarga/articulo/6336000.pdf

- Plá, S. (2016). Currículo, historia y justicia social. Estudio comparativo en América Latina. Revista colombiana de educación, (71), 53-77.

- Popkin, M. (2016). Iniciativas para mejorar la independencia judicia en América Latina: una perspectiva comparativa.

- Pring, C. (2017). Las personas y la corrupción: América Latina y el Caribe.

- Rivas, A. (2015). América Latina después de PISA: Lecciones aprendidas de la educación en siete países (2000-2015). Fundación Cippec.

- Rodríguez, I. C. R., Jaimes, E. C., \& Medina, J. E. C (s.f). Comprendiendo la pobreza: de la visión economicista a la complejidad multifactorial. Docencia, Ciencia y Tecnología.

- Rodríguez, J. G., \& Sánchez Riofrío, A. (2017). TIC y pobreza en América Latina.

- Rodríguez-Becerra, M., \& Espinoza, G. (2016). Gestión ambiental en América Latina y el Caribe: evolución, tendencias y principales prácticas.

- Rodríguez-Nava, A., \& Venegas-Martínez, F. (2014). Modelos actuales primario, secundario y terciario exportador en América Latina. Sección de Estudios de Posgrado e Investigación de la Escuela Superios de Economía del Instituto Politécnico Nacional, 1, 502-529.

- Roldán, I. G., \& Norato, O. M. G. (2014). ¿ Futuro limitado de la integración latinoamericana para impulsar el desarrollo?. Revista CIFE: Lecturas de Economía Social, 16(24), 195-215.

- Salama, P., \& Valier, J. (1995). Corrupción y pobreza. Espiral, 2(4), 45-67.Recuperado de: http://148.202.18.157/sitios/publicacionesite/pperiod/espiral/espiralpdf/espiral4/45-68.pdf

- Schmidtke, T., Koch, H., \& García, V. C. Los sectores económicos en América Latina.

- Sojo, A. (2011). Condiciones para el acceso universal a la salud en América Latina: derechos sociales, protección social y restricciones financieras y políticas. Ciência \& saúde coletiva, 16(6), 2673-2685.

- Soriano, R. (2011). La corrupción política: tipos, causas y remedios. In Anales de la Cátedra Francisco Suárez (Vol. 45, pp. 383-402).

- Stampini, M., Robles, M., Sáenz, M., Ibarrarán, P., \& Medellín, N. (2015). Pobreza, 
vulnerabilidad y la clase media en América Latina (No. IDB-WP-591). IDB Working Paper Series.

- Sutil, J. C. (2016). Acceso a la justicia y reformas judiciales en América Latina ¿ Alguna esperanza de mayor igualdad?.

- Vargas, G. I. D. L., León, D. M. B., \& Martinez, F. M. (2017). Amartya Sen, teoría de un desarrollo integral, aportes para la superación de la pobreza extrema y construcción de paz en los Montes de María.Erg@ omnes, 9(1),33-52.

- Vázquez Olivera, M. G. (2015). La calidad de la educación: Reformas educativas y control social en América Latina. Latinoamérica. Revista de Estudios Latinoamericanos, (60), 93124. 